\title{
O que a biologia não explica: grupos de afinidade no sambaqui Jabuticabeira II (Jaguaruna, SC)
}

\author{
Mercedes Okumura* \\ Sabine Eggers**
}

\begin{abstract}
OKUMURA, M.; EGGERS, S. O que a biologia não explica: grupos de afinidade no sambaqui Jabuticabeira II (Jaguaruna, SC). R. Museu Arq. Etn., São Paulo, 22: 97-109, 2012.
\end{abstract}

Resumo: A partir da análise da escavação do Locus 2 no sambaqui Jabuticabeira II, no sul de Santa Catarina, foi proposta a existência de um grupo de afinidade composto pelos indivíduos sepultados nesse local em um intervalo de tempo circunscrito. O objetivo do presente trabalho foi testar a hipótese de que os indivíduos do Locus 2 apresentam uma afinidade biológica maior entre si do que em relação aos demais indivíduos exumados de outros loci. Para tanto, dados craniométricos de 26 indivíduos foram analisados usando testes estatísticos. Nossos resultados não apoiam a hipótese de que os indivíduos do Locus 2 apresentem maior afinidade biológica entre si. À luz de dados da literatura, outros fatores biológicos também não corroboram a existência do grupo de afinidade. É possível que outros fatores, como os culturais, tenham influenciado a decisão de quem seria sepultado nesse Locus.

Palavras-chaves: Sambaqui, Craniometria, Jabuticabeira II, Pré-história brasileira

\section{Introdução}

Conhecidos desde o século XVI pelos colonizadores europeus, sambaquis são os sítios arqueológicos mais conspícuos na paisagem costeira brasileira. Embora diversos naturalistas e curiosos tenham citado a existência desses sítios, apenas no século XIX é que se iniciaram estudos de

(*) Museu de Arqueologia e Etnologia da Universidade de São Paulo. Pós-doutoranda em Arqueologia. <mercedes. okumura@gmail.com>

${ }^{(* *}$ Laboratório de Antropologia Biológica, Depto. de Genética e Biologia Evolutiva do Instituto de Biociências da Universidade de São Paulo. 〈saeggers@usp.br> natureza acadêmica para se entender a formação e demais características dos sambaquis.

Uma das características marcantes dos sambaquis é a presença, na maioria das vezes, abundante, de sepultamentos humanos. A grande quantidade de restos esqueletais exumados desses sítios possibilitou responder diversas questões acerca da biologia, do modo de vida, das origens e dispersões desses grupos costeiros (Gaspar, 1998; 2000).

A escavação do sambaqui Jabuticabeira II, datado entre 2880 e 1400 AP (De Blasis et al., 1998; Assunção, 2010; Giannini et al., 2010) forneceu informações importantes e permitiu a realização 
O que a biologia não explica: grupos de afinidade no sambaqui Jabuticabeira II (Jaguaruna, SC).

R. Museu Arq. Etn., São Paulo, n. 22: 97-109, 2012.

de diversos projetos de pesquisa tendo como foco áreas bastante distintas, desde antracologia (Bianchini et al., 2007; Bianchini, 2008), passando por geomorfologia (Giannini et al., 2010), estratigrafia (Villagran et al., 2010) e bioarqueologia.

Os restos esqueletais no Jabuticabeira II são abundantes, havendo evidências fortes de que o uso do sítio como cemitério estaria ligado ao seu processo de construção. Isso se daria através da preparação do local de sepultamento e da colocação de um montículo de sedimento misturado a conchas por cima dos locais onde os indivíduos eram sepultados (De Blasis et al., 1998; Fish et al., 2000; Gaspar et al., 1999; 2008; Klokler, 2008). Os estudos bioarqueológicos envolveram a análise do material esqueletal exumado do sítio a fim de responder diversas questões sobre a origem e afinidades biológicas (Filippini, 2004; Bartolomucci, 2006; Filippini \& Eggers, 2005-2006; Okumura, 2008), padrões de doenças (Storto et al., 1999;
Okumura \& Eggers, 2005; Petronilho, 2005; Okumura et al., 2007 a; b) e a subsistência desse grupo (Boyadjian, 2007; Boyadjian et al., 2007; Okumura \& Eggers, 2012).

Um dos locais de sepultamento que melhor foi caracterizado foi denominado de Locus 2 (Figura 1). Vale frisar que o termo original "Locus" foi mantido, pois não há tradução satisfatória para o português. Gaspar et al., (2008:325) descrevem esse local (tradução nossa): "uma escavação horizontal de $36 \mathrm{~m}^{2}$ dentro de uma área funerária confirmou que as camadas escuras eram superfícies de ocupação sucessivas, algumas vezes com pavimentos de conchas e sempre com inúmeros buracos de estaca nas vizinhanças dos sepultamentos. Os buracos de estaca rodeavam algumas covas funerárias e de modo semelhante demarcavam áreas funerárias inteiras. Estacas adicionais devem ter servido como suporte de estruturas pequenas colocadas sobre as covas (conforme observado em práticas

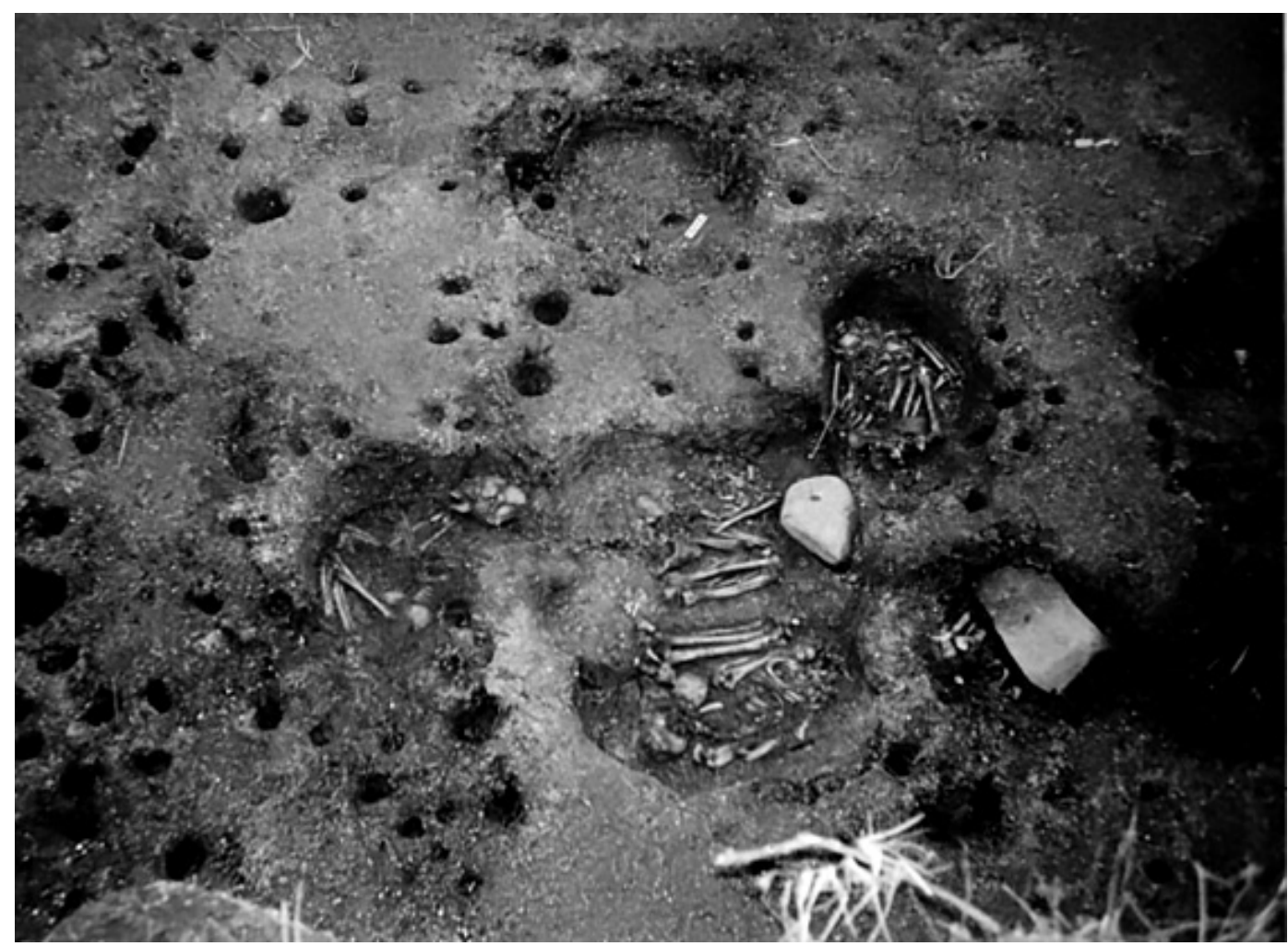

Fig. 1: Parte do Locus 2 com cinco indivíduos hiperfletidos evidenciados (dois cobertos por rochas) e um fundo de cova (no alto). Destaque para as marcas de estacas muito próximas e frequentemente delimitando os sepultamentos. 
etnográficas), como suporte de oferendas suspensas, assim como podem ter marcado as covas ou servido a outros propósitos."

Dessa forma, o Locus 2 têm sido considerado por alguns pesquisadores como o cemitério de um grupo de afinidade, isto é, um grupo de indivíduos que foi sepultado em uma área circunscrita e dentro de um período relativamente curto de tempo (Gaspar et al., 2008). Em termos temporais, ao menos dois indivíduos do Locus 2 apresentam datas não muito distantes, embora não se possa comprovar contemporaneidade. Um indivíduo da base da camada funerária e outro do topo (sendo que a camada funerária mede em torno de $25 \mathrm{~cm}$ de espessura) foram datados em $2.340 \pm 50$ e $2.320 \pm$ $50 \mathrm{AP}$, respectivamente (Beta 188381 and Beta 188382 - De Blasis et al., 2004). A calibracão resultaria em datas de 2465-2315 para o indivíduo da base da camada e 2370-2180 para o do topo (Bianchini, 2008:77).

Segundo Gaspar et al. (2008:324-325), áreas funerárias como o Locus 2 seriam interpretadas como locais designados para o enterramento de grupos de afinidade específicos, baseados em parentesco, filiação territorial ou outros princípios. A hipótese do grupo de afinidade do Locus 2 levantou questões sobre como essa afinidade poderia ser investigada. Uma das formas seria verificar a existência de um maior parentesco (traduzido em semelhanças morfológicas cranianas) entre os indivíduos do Locus 2 em relação aos demais. Este é o objetivo deste trabalho.

\section{Materiais e métodos}

O sexo dos indivíduos foi estimado segundo os critérios apresentados por Buikstra \& Ubelaker (1994) e White (1991). Neste estudo foram analisados apenas indivíduos adultos. A atribuição da idade de óbito em adultos foi realizada através da verificação da presença dos terceiros molares e do fechamento da sutura esfeno-occipital.

Caracteres métricos cranianos são variáveis contínuas obtidas a partir de medidas lineares (comprimentos, larguras, projeções ou rádios) que são usados para caracterizar o tamanho e a forma do crânio (Larsen, 1997). A tomada de medidas cranianas tem como objetivo implícito o estudo quantitativo do tamanho e da forma do crânio. Inúmeras tentativas foram feitas para se separar estes dois elementos (tamanho e forma), mas o problema é mais primário do que se pensa: não há uma definição única e precisa do que seja tamanho e do que seja forma. Assim, a forma não é uma medida individual que pode ser coletada diretamente, replicada ou conferida através da observação de um único organismo (Lele, 1991; Rao, 2000; Richsmeier et al., 2002). Apesar da linguagem usada em diversos estudos (incluindo este), a influência do fator tamanho nunca pode ser removida totalmente; e o tamanho e a forma nunca são biologicamente independentes; ao contrário, são intrinsecamente correlacionados. Ainda assim, diversos estudos baseados em medidas cranianas podem se beneficiar da tentativa de retirada parcial do fator tamanho, especialmente quando os grupos comparados possuem diferenças marcantes em relação a esse aspecto. Nesses casos, se o fator tamanho não for removido, as diferenças entre os grupos envolvidos deverão ser, na sua maioria, produzidas pelo tamanho.

É inegável a eficiência do uso de elementos craniodentais pata testar hipóteses acerca de filogenias e taxonomias. Esta abordagem reflete parcialmente a frequente preservação e fácil identificação de restos cranianos e dentários, assim como a premissa que a morfologia craniana é um bom indicador das relações biológicas entre ancestrais e descendentes.

Foram excluídas as variáveis e os espécimes cujos valores faltantes excediam $50 \%$. Tal procedimento reduziu o número de variáveis craniométricas a apenas seis, no entanto, tais variáveis conseguem descrever de maneira satisfatória as principais dimensões do crânio. Os valores ausentes remanescentes foram substituídos pelas médias entre todos os grupos. As seis variáveis craniométricas utilizadas foram descritas por Howells $(1973,1989)$. Os nomes e as siglas foram mantidos em inglês, tendo em vista seu amplo emprego pela comunidade bioantropológica internacional. São elas (Figura 2): 
GOL (Glabello-occipital lenght) - Comprimento da glabela ao opistocranium, no plano médio sagital.

STB (Bistephanic breadth) - Largura entre as intersecções da sutura coronal e da linha temporal inferior.

FMB (Bifrontal breadth) - Largura do osso frontal entre os frontomalares anteriores.

DKB (Interorbital breadth) - Largura do espaço nasal medida entre os dacryons.

WMH (Minimum malar height) - Em qualquer direção, da borda inferior da órbita até a margem inferior da maxila.

FMR (Frontomalare anterior radius) - Distância perpendicular entre o eixo transmeatal e o frontomalare esquerdo.

A Tabela 1 apresenta a amostra de indivíduos analisada. Foram estudados 26 indivíduos, sendo sete oriundos do Locus 2 e 19 provenientes de outros loci do sítio, que foram renomeados Locus 1 (vide coluna "Locus final”) a fim de facilitar as análises. Devido à exiguidade da amostra, indivíduos masculinos e femininos foram analisados em conjunto.
Foi realizada a correção de tamanho dos crânios através do método da média geométrica (Darroch \& Mosimann, 1985). Tal método divide o valor de cada uma das variáveis pela média geométrica de todas as variáveis de cada indivíduo ou amostra. A adoção desta correção é necessária a fim de que a forma do crânio, e não o tamanho do mesmo, seja a responsável pelas diferenças morfológicas encontradas entre os grupos analisados.

Foram realizadas três análises estatísticas: Análise de Componentes Principais, Análise de Funções Discriminantes e Teste T. A Análise de Componentes Principais (a partir de matrizes de covariância) tem como objetivo explorar as correlações entre um grande número de variáveis quantitativas inter-relacionadas através do agrupamento dessas variáveis em uns poucos fatores ou componentes independentes entre si. A análise de Funções Discriminantes tem por objetivo locar indivíduos isolados em grupos de referência, a partir de um conjunto de variáveis fornecidas. As medidas de cada indivíduo são
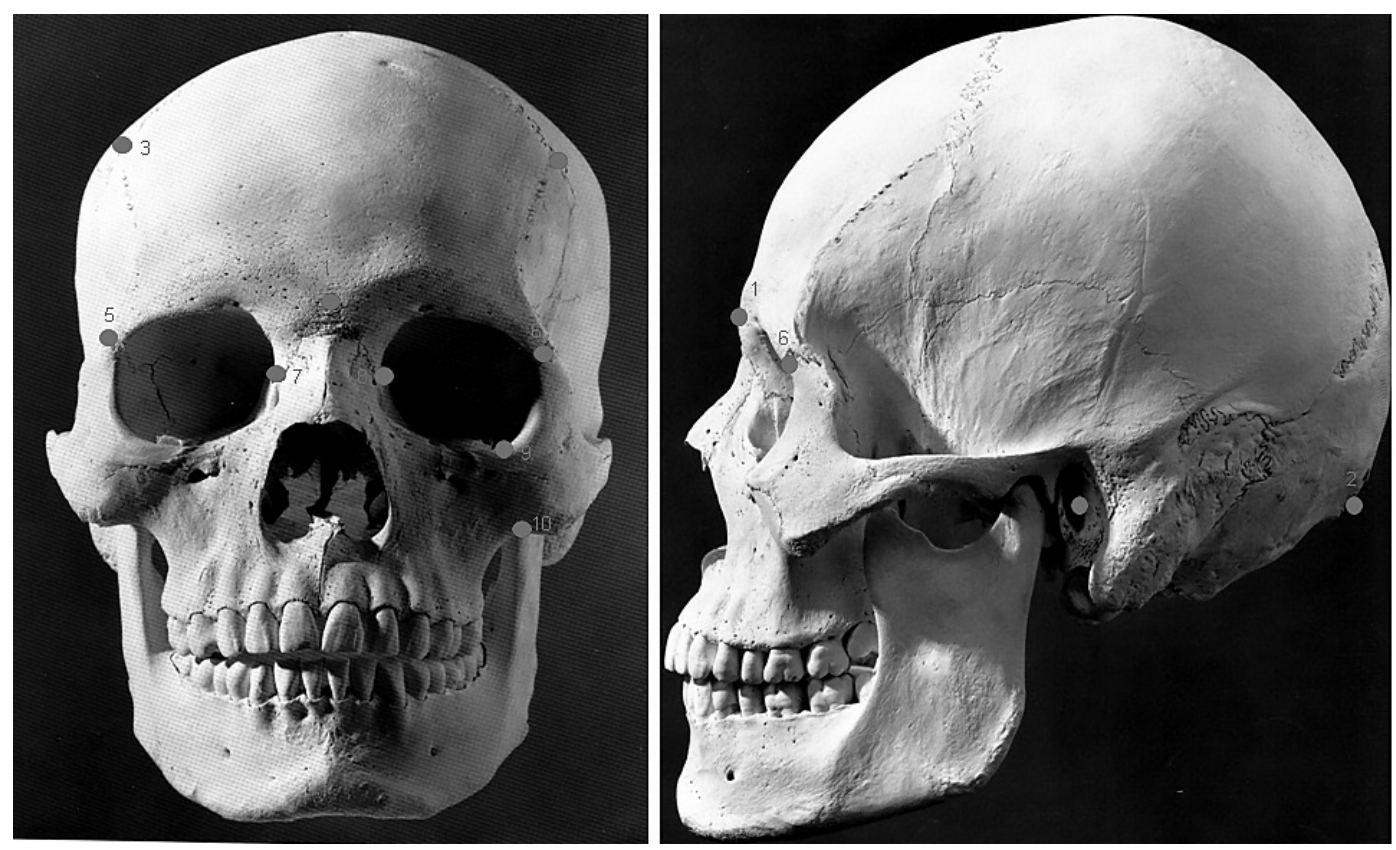

Fig. 2: As seis variáveis craniométricas utilizadas na análise. Os números referem-se aos pontos de referência a partir dos quais as medidas são tiradas. GOL (1-2). STB (3-4), FMB (5-6), DKB (7-8), WMH (9-10), FMR (6-11). Fotos de White (1991). 


\begin{tabular}{|c|c|c|c|}
\hline Identificação & Locus & Locus final & Sexo \\
\hline Sep11/E1/L1.25 & 1.25 & 1 & masculino \\
\hline Sep3B2/E1/L1.05 & 1.05 & 1 & masculino \\
\hline Sep6/E1/L1.10 & 1.1 & 1 & masculino \\
\hline Sep10B/E1/L1.25 & 1.25 & 1 & masculino \\
\hline Sep12C/E1/L1.25 & 1.25 & 1 & masculino \\
\hline Sep15/E1/L1.05 & 1.05 & 1 & masculino \\
\hline Sep17A/E1/L1.05 & 1.05 & 1 & masculino \\
\hline Sep1B/E2/L6C & $6 \mathrm{C}$ & 1 & feminino \\
\hline Sep2A/E3/L6 & 6 & 1 & masculino \\
\hline Sep42/E3/L1.76 & 1.76 & 1 & feminino \\
\hline Sep43/E3/L1.77 & 1.77 & 1 & masculino \\
\hline Sep107/E5/L1.T18 & $1 / \mathrm{T} 18$ & 1 & masculino \\
\hline Sep 3B/L6/QB3/FS2111 & 6 & 1 & feminino \\
\hline Sep 107/L1/T18/E5 & 1 & 1 & masculino \\
\hline Sep 111/112 Pat/Perfil E/TL1/L2 & TL1/L2 & 1 & masculino \\
\hline Sep 111/112 não Pat/Perfil E/TL1/L2 & TL1/L2 & 1 & masculino \\
\hline Sep 115B/L6/FS2136 & 6 & 1 & masculino \\
\hline Sep 118/L6/Camada 2 & 6 & 1 & masculino \\
\hline Sep 121/L6/FS2138 & 6 & 1 & masculino \\
\hline Sep17/E3/L2.05PD & 2.05 & 2 & masculino \\
\hline Sep25/E3/L2.65 & 2.65 & 2 & feminino \\
\hline Sep41A/E4/L2.05 & 2.05 & 2 & masculino \\
\hline Sep 34/L2.05/E4 & 2.05 & 2 & feminino \\
\hline Sep 36A/L2.05/E4 & 2.05 & 2 & masculino \\
\hline Sep 37/E4/L2.05 & 2.05 & 2 & masculino \\
\hline Sep37/E4 & 2 & 2 & masculino \\
\hline
\end{tabular}

Tabela 1: Descrição da amostra, locus de origem e sexo estimado.

comparadas com os centroides de cada série, levando-se em consideração tanto a variância das séries quanto o número de indivíduos em cada uma delas. A variância é utilizada para estabelecer, dentro de cada série, o limiar de dispersão dos valores observados de cada variável. Desta forma, cada indivíduo possui uma probabilidade diferente de classificação em cada uma das séries incluídas na análise. Uma vez que um indivíduo possui maior probabilidade de ser classificado nas séries maiores devido ao acaso, as probabilidades obtidas são ponderadas de acordo com o número de indivíduos de cada série (Albrecht, 1992; Van Vark \& Schasma, 1992). Finalmente, o Teste $T$ verifica se a média de uma variável em um grupo é significativamente diferente da média observada no outro grupo (Madrigal, 1998:96). 
As análises estatísticas foram realizadas com os softwares Statistica e SPSS.

\section{Resultados}

A Tabela 2 apresenta as principais características dos componentes principais gerados pela Análise de Componentes Principais. Conforme pode ser visto, os dois primeiros autovalores explicam $83,8 \%$ da variância total.

A Figura 3 apresenta o morfoespaço formado pelos dois primeiros Componentes Principais. Não é possível verificar nenhuma separação clara entre os indivíduos do Loci 1 e 2, tampouco pode-se observar alguma região no gráfico onde exista uma concentração de indivíduos de um ou outro locus.

A Tabela 3 apresenta os resultados da Análise Discriminante. Pode-se verificar que enquanto os indivíduos do Locus 1 apresentam uma alta porcentagem de classificação correta em seu grupo, os do Locus 2 não mostram o mesmo resultado.

A Tabela 4 mostra as probabilidades de classificação dos indivíduos no Loci 1 e 2. Verifica-se que os indivíduos do Locus 2 erroneamente classificados como sendo do Locus 1 , apresentam probabilidades relativamente altas de pertencer ao grupo do Locus 1 .
Finalmente, foi realizado um Teste T. A Tabela 5 apresenta as médias e desvios-padrão para cada variável nos dois loci.

A Tabela 6 apresenta o Teste de Levene, que deve ser feito antes do Teste $T$ para testar se as variâncias dos dois grupos são iguais. Dado que os valores de $\mathrm{p}$ não são significativos (coluna "p de Levene"), é possível assumir variâncias iguais para os dois grupos. Os valores de $\mathrm{p}$ do Teste $\mathrm{T}$ mostram que não há diferenças significativas entre as médias das variáveis dos dois grupos.

\section{Discussão e conclusões}

Nossos resultados mostram que não há diferenças significativas entre o suposto grupo de afinidade (indivíduos do Locus 2) e os indivíduos exumados em outros loci do sítio Jabuticabeira II. Ou seja, não existe nenhuma afinidade maior entre indivíduos do Locus $2 \mathrm{em}$ relação aos demais indivíduos sepultados nesse sambaqui.

No entanto, é necessário levar em conta as fragilidades inerentes às análises aqui apresentadas. Um dos principais problemas foi o pequeno tamanho amostral, que não apenas enfraquece as análises estatísticas como também fez com que tivéssemos que analisar ambos os sexos em

\begin{tabular}{|c|c|c|c|c|}
\hline & Autovalor & $\begin{array}{c}\text { \% total da } \\
\text { variância }\end{array}$ & $\begin{array}{c}\text { Autovalor acu- } \\
\text { mulado }\end{array}$ & \% acumulada \\
\hline 1 & 0,010790 & 67,49678 & 0,010790 & 67,4968 \\
\hline 2 & 0,002606 & 16,29814 & 0,013396 & 83,7949 \\
\hline 3 & 0,001771 & 11,08097 & 0,015167 & 94,8759 \\
\hline 4 & 0,000691 & 4,32128 & 0,015858 & 99,1972 \\
\hline 5 & 0,000127 & 0,79441 & 0,015985 & 99,9916 \\
\hline 6 & 0,000001 & 0,00841 & 0,015987 & 100 \\
\hline
\end{tabular}

Tabela 2: Autovalores, porcentagem total da variância explicada por cada autovalor, autovalores acumulados e porcentagens acumuladas de cada autovalor. 


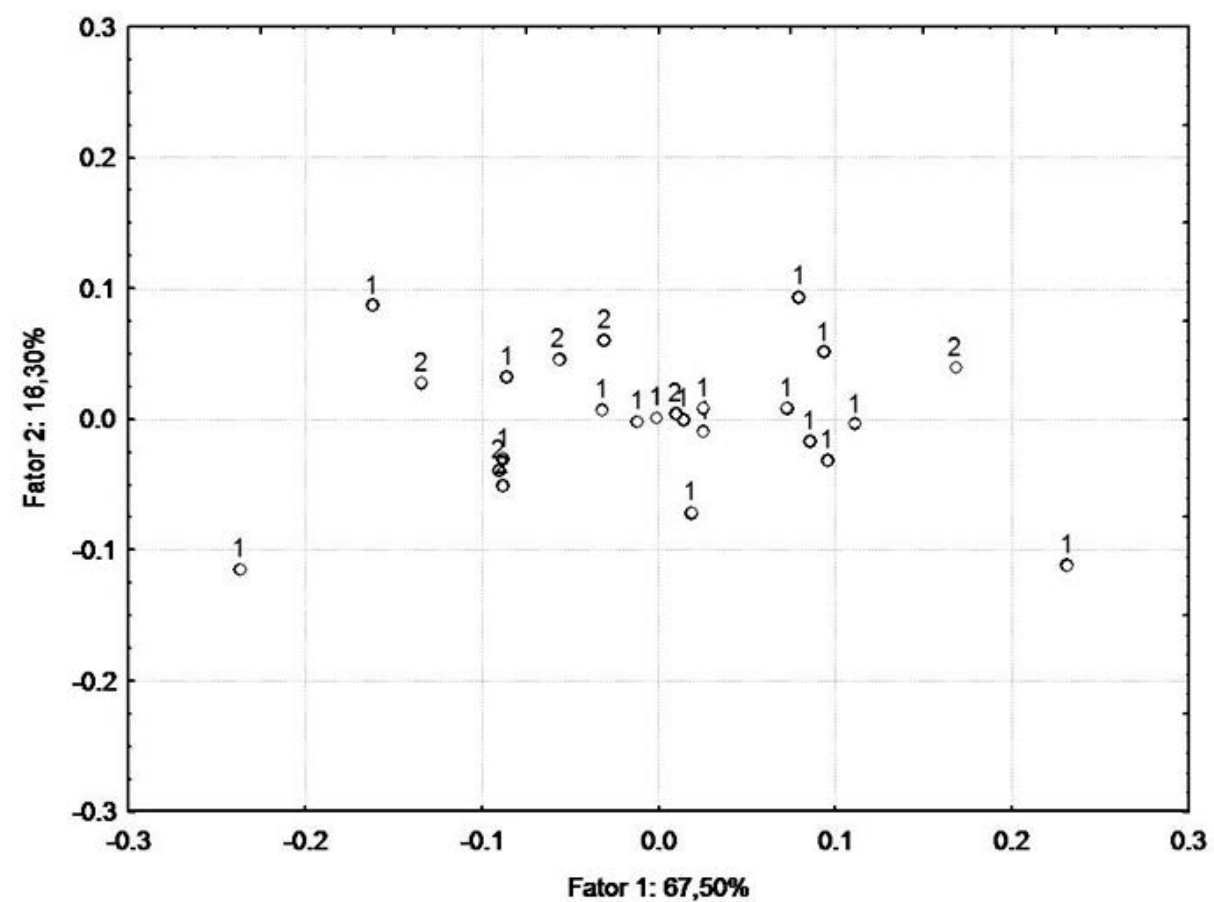

Fig. 3: Dispersão dos indivíduos do Loci 1 e 2 no morfoespaço formado pelos dois primeiros Componentes Principais.

\begin{tabular}{|c|c|c|c|}
\hline & $\begin{array}{c}\text { \% de classificação cor- } \\
\text { reta }\end{array}$ & $\begin{array}{c}\text { Número de indivíduos clas- } \\
\text { sificados no Locus 1 }\end{array}$ & $\begin{array}{c}\text { Número de indivíduos classi- } \\
\text { ficados no Locus 2 }\end{array}$ \\
\hline Locus 1 & 94,74 & 18 & 1 \\
\hline Locus 2 & 28,57 & 5 & 2 \\
\hline Total & 76,91 & 23 & 3 \\
\hline
\end{tabular}

Tabela 4: Probabilidades de classificação dos indivíduos no Loci 1 e 2. Asterisco indica classificação equivocada.

conjunto. No entanto, análises que levaram em conta apenas os indivíduos do sexo masculino apresentaram resultados muito semelhantes (os resultados dessa análise não foram mostrados neste artigo). A substituição dos valores faltantes pela média regredida da amostra total também ajuda a homogeneizar a amostra. No entanto, tal procedimento teve que ser feito para se obter um número minimamente aceitável de variáveis e de indivíduos. A desigualdade do número de indivíduos nos dois grupos analisados através da Análise Discriminante pode ter mascarado possíveis diferenças morfológicas. É sabido que geralmente o grupo com o maior número de indivíduos é o que consegue "classificar" mais indivíduos. No entanto, o fato dos indivíduos do Locus 2 erroneamente classificados como sendo do Locus 1 terem apresentado probabilidades relativamente altas de classificação indica que é possivel aceitar que de fato não há diferenças importantes entre os dois grupos. Outra limitação do presente trabalho é o número reduzido de medidas que puderam ser tomadas de todos os crânios para salvaguardar a comparabilidade. Isso é consequência da ausência de diferentes fragmentos cranianos, decorrente de problemas de preservação e conservação do material osteológico. Entretanto, as variáveis incluídas nas 


\begin{tabular}{|c|c|c|}
\hline Locus & $\begin{array}{c}\text { Probabilidade de classificação no } \\
\text { Locus } 1\end{array}$ & $\begin{array}{c}\text { Probabilidade de classificação no } \\
\text { Locus } 2\end{array}$ \\
\hline 1 & 0,69 & 0,31 \\
\hline 1 & 0,61 & 0,39 \\
\hline 1 & 0,68 & 0,32 \\
\hline 1 & 0,88 & 0,12 \\
\hline$* 1$ & 0,35 & 0,65 \\
\hline 1 & 0,82 & 0,18 \\
\hline 1 & 0,91 & 0,09 \\
\hline 1 & 0,74 & 0,26 \\
\hline 1 & 0,69 & 0,31 \\
\hline 1 & 0,53 & 0,47 \\
\hline 1 & 0,81 & 0,19 \\
\hline 1 & 0,94 & 0,06 \\
\hline 1 & 0,68 & 0,32 \\
\hline 1 & 0,96 & 0,04 \\
\hline 1 & 0,84 & 0,16 \\
\hline 1 & 0,75 & 0,25 \\
\hline 1 & 0,96 & 0,04 \\
\hline 1 & 0,92 & 0,08 \\
\hline 1 & 0,95 & 0,05 \\
\hline$* 2$ & 0,74 & 0,26 \\
\hline 2 & 0,42 & 0,58 \\
\hline 2 & 0,32 & 0,68 \\
\hline *2 & 0,67 & 0,33 \\
\hline$* 2$ & 0,77 & 0,23 \\
\hline$* 2$ & 0,68 & 0,32 \\
\hline$* 2$ & 0,67 & 0,33 \\
\hline
\end{tabular}

Tabela 4: Probabilidades de classificação dos indivíduos no Loci 1 e 2. Asterisco indica classificação equivocada.

análises representam as principais dimensões cranianas, sendo um modo satisfatório de descrever e comparar esses crânios.

Com base nos nossos resultados, não é possível afirmar que os indivíduos sepultados no Locus 2 sejam mais semelhantes entre si em termos de morfologia craniana em relação aos demais indivíduos do mesmo sítio. Mesmo que esta análise apresente algumas limitações, nossos resultados corroboram outros estudos que também não detectaram diferenças entre os indivíduos do Locus 2 em relação aos demais.

O estudo realizado por Bartolomucci (2006) comparou os indivíduos do Locus 2 com outros indivíduos de outros loci do Jabuticabeira II através da análise de caracteres não 


\begin{tabular}{|c|c|c|c|}
\hline & Locus & Média & Desvio-padrão \\
\hline GOL & 1 & 2,5392 & 0,0884 \\
\hline & 2 & 2,506 & 0,0845 \\
\hline STB & 1 & 1,5777 & 0,0508 \\
\hline & 2 & 1,5704 & 0,0511 \\
\hline FMB & 1 & 1,4069 & 0,0406 \\
\hline & 2 & 1,3915 & 0,0403 \\
\hline DKB & 1 & 0,3186 & 0,0238 \\
\hline & 2 & 0,3274 & 0,0196 \\
\hline WMH & 1 & 0,3505 & 0,0134 \\
\hline & 2 & 0,3578 & 0,0104 \\
\hline FRC & 1 & 1,5982 & 0,0652 \\
\hline & 2 & 1,5643 & 0,0374 \\
\hline
\end{tabular}

Tabela 5: Média e desvio-padrão das seis variáveis para os dois loci.

\begin{tabular}{|c|c|c|c|c|c|}
\hline Variável & $\begin{array}{c}\text { Teste de } \\
\text { Levene }\end{array}$ & $\begin{array}{c}\mathbf{p} \text { de } \\
\text { Levene }\end{array}$ & Teste T & $\begin{array}{c}\text { Graus de } \\
\text { liberdade }\end{array}$ & $\begin{array}{c}\mathbf{p} \text { do } \\
\text { Teste T }\end{array}$ \\
\hline GOL & 0.004 & 0.951 & 0.858 & 24 & 0.399 \\
\hline STB & 0.132 & 0.719 & 0.323 & 24 & 0.749 \\
\hline FMB & 0.085 & 0.773 & 0.861 & 24 & 0.398 \\
\hline DKB & 0.389 & 0.539 & -0.88 & 24 & 0.388 \\
\hline WMH & 0.681 & 0.417 & -1.296 & 24 & 0.207 \\
\hline FRC & 1.238 & 0.277 & 1.288 & 24 & 0.21 \\
\hline
\end{tabular}

Tabela 6: Teste de Levene e Teste $\mathrm{T}$.

métricos dentários, sem encontrar diferenças significativas. Da mesma forma, Filippini (2004) também não observou nenhuma afinidade maior entre os indivíduos do Locus 2 ao estudar caracteres não métricos cranianos. Em termos de padrões e frequências de osteo-artroses, também parece não haver diferenças importantes quanto à intensidade e/ou padrão de atividade física entre os indivíduos do Locus 2 e os demais (Petronilho, 2005).

Além disso, foram realizados estudos com a finalidade de melhor compreender se a dieta do grupo de afinidade do Locus 2 poderia refletir um acesso diferencial a recursos. Oito indivíduos de Jabuticabeira II foram submetidos a estudos de isótopos estáveis de carbono e nitrogênio a partir do colágeno, fornecendo dados sobre o aporte proteico (Richards et al., 2007). Os quatro indivíduos do suposto grupo de afinidade do Locus 2 (dois homens e duas mulheres) amostrados nesse trabalho apresentam menor variabilidade quanto aos dois marcadores, além de uma média maior de $\mathrm{d} 15 \mathrm{~N}$, sugerindo uma dieta mais rica em proteínas em relação aos de- 
mais indivíduos de Jabuticabeira II. Entretanto, Klokler (2008), ao incluir mais oito indivíduos na amostra, não observa diferenças importantes na dieta. Valendo-se de análises zooarqueológicas, os alimentos oferecidos durante rituais funerários em 12 supostos grupos de afinidade de Jabuticabeira II também não puderam ser discriminados (Klokler, 2008). Da mesma maneira, a avaliação de microrrestos vegetais extraídos do cálculo dentário não revelou que os indivíduos do grupo de afinidade do Locus 2 tenham um consumo de vegetais muito distinto em comparação aos indivíduos sepultados em outras localidades do sítio (Boyadjian, 2012). Dessa maneira, nenhuma das sete abordagens utilizadas (craniometria, variáveis não métricas cranianas e dentárias, estudos zooarqueológicos, análise de isótopos estáveis, padrões e frequências de osteo-artrose e a análise de microrrestos vegetais do cálculo dentário) foi capaz de encontrar evidências sobre a existência de diferenças entre os supostos grupos de afinidade em Jabuticabeira II.

Finalmente, é importante frisar a atuação de pesquisadores de diversas subáreas da arqueologia na proposição de hipóteses que podem ser testadas através do estudo de restos esqueletais humanos. Certamente, esses estudos podem se beneficiar da exploração de problemas arqueológicos propostos por acadêmicos cujo olhar para questões afins seja diferente do nosso.

\section{Agradecimentos}

Este artigo é uma versão estendida de uma apresentação feita pelas autoras no XVI Congresso Mundial da UISPP e no XVI Congresso da SAB que ocorreu em setembro de $2011 \mathrm{em}$ Florianópolis. Agradecemos a todos que contribuíram para a discussão, especialmente a Maria Dulce Gaspar pela proposição da hipótese dos grupos de afinidade. Agradecemos também aos coordenadores da escavação do Jabuticabeira II: Maria Dulce Gaspar, Paulo De Blasis, Paul Fish e Suzane Fish, e a Cecilia Petronilho pelo auxílio técnico. Esta pesquisa foi financiada pela FAPESP (processo 02/13441-0). Sabine Eggers é bolsista de produtividade do CNPq.

OKUMURA, M.; EGGERS, S. What biology cannot explain: affinity groups from the shellmound Jabuticabeira II (Jaguaruna, SC). R. Museu Arq. Etn., São Paulo, 22: 97-109, 2012.

Abstract: The excavation of Locus 2 in the shellmound Jabuticabeira II, south of Santa Catarina resulted in the proposal of the existence of an affinity group made up of individuals buried in this place in a limited time interval. The aim of this study was to test the hypothesis that individuals from Locus 2 are more closely related in biological terms than in relation to individuals exhumed from other loci. Craniometric data from 26 individuals were studied using statistical analysis. Our results do not support the hypothesis that individuals from Locus 2 have a greater biological affinity between them. Since other biological criteria were also excluded, it is possible that other factors (possibly cultural ones) would have influenced the criteria of who would be buried at this locus.

Keywords: Shellmound, Craniometrics, Jabuticabeira II, Brazilian prehistory 


\section{Referências Bibliográficas}

ALBRECHT, G. H.

1992 Assessing the affinities of fossils using canonical variates and generalized distances. Human Evolution 7(4):49-69.

ASSUNÇÃO, D. C.

2010 Sambaquis da paleolaguna de Santa Marta: em busca do contexto regional no litoral sul de Santa Catarina. Dissertação de Mestrado. São Paulo: Museu de Arqueologia e Etnologia - USP. 146 p.

BARTOLOMUCCI, L. B. G.

2006 Variabilidade Biológica entre Sambaquieiros: um estudo de morfologia dentária. Dissertação de Mestrado. São Paulo: Departamento de Genética e Biologia Evolutiva - USP. 105 p.

BIANCHINI, G. F.; SCHEEL-YBERT, R.; GASPAR, M. D.

2007 Estaca de Lauraceae em contexto funerário (sítio Jaboticabeira-II, Santa Catarina, Brasil). Revista do Museu de Arqueologia e Etnologia 17: 223-229.

BIANCHINI, G. F.

2008 Fogo e Paisagem: evidências de práticas rituais e construção do ambiente a partir da análise antracológica de um sambaqui no litoral sul de Santa Catarina. Dissertação de Mestrado. Rio de Janeiro: Universidade Federal do Rio de Janeiro (UFRJ). 200 p.

BOYADJIAN, C. H. C.

2007 Microfósseis contidos no cálculo dentário como evidência do uso de recursos vegetais os sambaquis de Jabuticabeira II (SC) e Moraes (SP). Dissertação de Mestrado. São Paulo: Departamento de Genética e Biologia Evolutiva - USP. 147 p.

BOYADJIAN, C. H. C.; EGGERS, S.; REINHARD, K. J.

2007 Dental wash: a problematic method for extracting microfossils from teeth. Journal of Archaeological Science 34: 1622-1628.

BOYADJIAN, C. H. C.

2012 Análise e identificação de microvestígios vegetais de cálculo dentário para a reconstrução de dieta sambaquieira: estudo de caso de Jabuticabeira II, SC. Tese de Doutorado. São Paulo: Departamento de Genética e Biologia Evolutiva - USP. 226 p.

BUIKSTRA, J. E.; UBELAKER, D. H.

1994 Standards for Data Collection from Human Skeletal Remains. Fayetteville: Arkansas
Archaeological Survey Research Series ${ }^{\circ}$ 44. $272 \mathrm{p}$

DARROCH, J. N.; MOSIMANN, J. E.

1985 Canonical and principal components of shape. Biometrika 72: 241-252.

DE BLASIS P. A. D.; FISH, S. K.; GASPAR, M. D.; FISH, P. R.

1998 Some references for the discussion of complexity among the sambaqui mound builders from the southern shores of Brazil. Revista de Arqueología Americana 15: 75-105.

DE BLASIS, P. A. D.; GASPAR, M. D.; GIANNINI, P. C. F.; FIGUTI, L.; EGGERS, S.; SCHEEL-YBERT, R.; AFONSO, M. C.; FARIAS, D. S.; KNEIP, A.; MENDONÇA, C. A.; YBERT, J.-P.; KLÖKLER, D. M.; SAWAKUSHI, A.; BARBOSA, P. N.; BENDAZZOLI,

C. DE SOUZA; J. B.; RIBEIRO, F. V. A.; ARIZA, M.

2004 Processos Formativos nos Sambaquis do Camacho, SC: Padrões Funerários e Atividades Cotidianas. Relatório Final. São Paulo: Fapesp (processo: 03/02059-0)

FILIPPINI, J.

2004 Biodistância entre sambaquieiros fluviais e costeiros. Uma abordagem não-métrica craniana entre três sítios fluviais do Vale do Ribeira - SP (Moraes, Capelinha e Pavão XVI) e três costeiros do sul e sudeste do Brasil (Piaçagüera, Jabuticabeira II e Tenório). São Paulo: Museu de Arqueologia e Etnologia - USP. $247 \mathrm{p}$.

FILIPPINI, J.; EGGERS, S.

2005/2006 Distância Biológica entre Sambaquieiros Fluviais (Moraes - Vale do Ribeira - SP) e Construtores de Sítios Litorâneos (Piaçaguera e Tenório-SP e Jabuticabeira II-SC). Revista do Museu de Arqueologia e Etnologia 15-16: 165-180.

FISH, S. K.; DE BLASIS, P. A. D.; GASPAR, M. D.; FISH, P. R.

2000 Eventos Incrementais na Construção de Sambaquis, Litoral Sul do Estado de Santa Catarina. Revista do Museu de Arqueologia e Etnologia 10: 69-87.

GASPAR, M. D

1998 Considerations of the sambaquis of the Brazilian coast. Antiquity 72:592-615.

GASPAR, M. D.; AFONSO, M. C.; DE BLASIS, P. A. D.; EGGERS, S.; FIGUTI, L.; FISH, S. K.; FISH, P. R.; KLÖKLER, D. M.; LAHR, M. M.; MOELEY, E. J. 1999 Uma Breve História do Projeto de Pesquisa "Padrão de Assentamento e Formação 
de Sambaquis: Arqueologia e Preservação em Santa Catarina”. Revista do Centro de Estudos e Pesquisas de Arqueologia (CEPA) 23(29):103-117.

GASPAR, M. D.; DE BLASIS, P. A. D.; FISH, S. K.; FISH, P. R.

2008 Sambaqui (Shell Mound) Societies of Coastal Brazil. In: Silverman H, and Isbell WH, editors. Handbook of South American Archaeology. New York: Springer. p 319-335.

GIANINI, P. C. F.; VILAGRAN, X. S.; FORNARI, M.; DO NASCIMENTO JR., D. R.; MENEZES, P. M. L.; TANAKA, A. P. B.; ASSUNÇÃO, D. C.; DE BLASIS, P.; DO AMARAL, P. G. C.

2010 Interações entre evolução sedimentar e ocupação humana pré-histórica na costa centro-sul de Santa Catarina, Brasil. Boletim do Museu Paraense Emílio Goeldi Ciências Humanas 15(1): 105-128.

HOWELLS, W. W.

1973 Cranial Variation in Man: A Study by Multivariate Analysis of Patterns of Difference Among Recent Human Populations. Papers of the Peabody Museum of Archaeology and Ethnology, v. 67. Cambridge, MA:

HOWELLS, W. W. Harvard University Press. 259 p.

1989 Skull Shapes and the Map: Craniometric Analyses in the Dispersion of Modern Homo. Papers of the Peabody Museum of Archaeology and Ethnology, v. 79 Cambridge,

KLÖKLER, D. M. MA Harvard University Press. 187 p.

2008 Food for body and soul: Mortuary ritual in shell mounds (Laguna-Brazil). PhD thesis. Arizona: University of Arizona. 369 p.

LARSEN, C. S.

1997 Bioarchaeology: interpreting behavior from human skeleton. Cambridge: Cambridge University Press. 461 p.

LELE, S.

1991 Some comments on coordinate-free and scale-invariant methods in morphometrics. American Journal of Physical Anthropology 85(4): 407-417.

MADRIGAL, L.

1998 Statistics for Anthropology. Cambridge: Cambridge University Press. 238 p.

OKUMURA, M. M. M.

2008 Diversidade morfológica craniana, microevolução e ocupação pré-histórica da costa brasileira. Pesquisas Antropologia 66.

OKUMURA, M. M.; EGGERS, S.

2005 The people of Jabuticabeira II: recons- truction of the way of life in a Brazilian shellmound. Homo 55(3): 263-281.

OKUMURA, M. M.; BOYADJIAN, C. H., EGGERS, S.

2007 Auditory exostoses as an aquatic activity marker: a comparison of coastal and inland skeletal remains from tropical and subtropical regions of Brazil. American Journal of Physical Anthropology 132(4): 558-567.

OKUMURA, M. M.; BOYADJIAN, C. H.; EGGERS, S. 2007 An evaluation of auditory exostoses in 621 prehistoric human skulls from coastal Brazil. Ear, Nose, $\mathcal{E}$ Throat Journal 86(8):468-472.

OKUMURA, M. M.; EGGERS, S.

2008 Natural and Cultural Formation Processes on the Archaeological Record: A Case Study regarding Skeletal Remains from a Brazilian Shellmound. In: Suárez AR, and Vásquez MN, editors. Archeology Research Trends. Hauppauge, NY: Nova Science Publishers. p 1-39.

OKUMURA, M. M.; EGGERS, S.

2012 Living and eating in coastal southern Brazil during Prehistory: a review. In: Collard D, Morris J, and Perego E, editors. Food $\mathcal{B}$ Drink in Archaeology 3. Devon: Prospect Books. p 55-64.

PETRONILHO, C. C.

2005 Comprometimento articular como um marcador de atividades em um grande Sambaqui-Cemitério. Dissertação de Mestrado. São Paulo: Departamento de Genética e Biologia Evolutiva - USP. 176 p.

$\mathrm{RAO}, \mathrm{C}$.

2000 A note on statistical analysis of shape through triangulation of landmarks. Proc Natl Acad Sci USA 97: 2995-2998.

RICHARDS, M.; DE BLASIS, P. A. D.; EGGERS, S.

2007 Stable Isotopes and what they reveal about paleodiet in Jabuticabeira II. XIV Congresso da Sociedade de Arqueologia Brasileira, Florianópolis. Simpósio "Bioarqueologia de Sambaquis".

RICHTSMEIER, J. T.; DELEON, V. B. LELE, S. R.

2002 The Promise of Geometric Morphometrics. Yearbook Of Physical Anthropology 45: 63-91.

STORTO, C.; EGGERS, S.; LAHR, M. M.

1999 Estudo preliminar das paleopatologias da população do sambaqui Jaboticabeira II, Jaguaruna, SC. Revista do Museu de Arqueologia e Etnologia 9: 61-71. 
VAN VARK, G. N.; SCHAFSMA, W.

1992 Advances in quantitative analysis of skeletal morphology. In: Saunders SR, Katzenberg A, editors. Skeletal biology of past people: research methods. New York: Willey-Liss.

VILLAGRAN, X. S.; KLÖKLER, D. M.; NISHIDA, P. GASPAR, M. D.; DE BLASIS, P. A. D.
2010 Lecturas Estratigráficas: Arquitectura Funeraria y Depositación de Residuos en el Sambaquí Jabuticabeira II. Latin American Antiquity 21(2):195-216.

WHITE, T. D.

1991 Human Osteology. San Diego: Academic Press. 455 p. 